\title{
Labyrinthe
}

39 | $2012(2)$

"Et si... ? " La cause du contrefactuel

\section{Contrefactuels en histoire : du mot au mode d'emploi. Le moment de la new economic history}

Isabelle Drouet, Stéphanie Dupouy, Laurent Jeanpierre et Florian Nicodème

\section{(2) OpenEdition}

\section{Édition électronique}

URL : http://journals.openedition.org/labyrinthe/4272

DOI : $10.4000 /$ labyrinthe.4272

ISSN : 1950-6031

Éditeur

Hermann

\section{Édition imprimée}

Date de publication : 31 décembre 2012

Pagination : 81-112

ISBN : 9782705682637

\section{Référence électronique}

Isabelle Drouet, Stéphanie Dupouy, Laurent Jeanpierre et Florian Nicodème, "Contrefactuels en histoire : du mot au mode d'emploi. Le moment de la new economic history ", Labyrinthe [En ligne], 39 | 2012 (2), mis en ligne le 10 janvier 2013, consulté le 20 avril 2019. URL : http://

journals.openedition.org/labyrinthe/4272 ; DOI : 10.4000/labyrinthe.4272 


\title{
Contrefactuels en histoire : du mot au mode d'emploi. Le moment de la new economic history
}

\author{
Isabelle DrouET, Université Paris IV \\ Stéphanie Dupouy, Université de Strasbourg \\ Laurent JeANPIERRE, Université Paris VIII - Saint-Denis \\ Florian NicodÈME, ENS-Ulm
}

L'approche contrefactuelle désigne, pour les historiens, une forme particulière que peut prendre le récit historique ou la pratique de l'histoire. Raisonner contrefactuellement, dans ce cadre, c'est se demander ce qui se serait passé si la réalité avait été différente. Le plus souvent, il s'agit de supprimer en pensée un événement ou une réalité historique et de s'interroger sur les conséquences de cette suppression. Que se seraitil passé si Hitler était mort pendant la Première Guerre mondiale, ou encore si la Peste n'avait pas décimé l'Europe à plusieurs reprises entre le $\mathrm{vI}^{\mathrm{e}}$ et le $\mathrm{VIII}^{\mathrm{e}}$ siècle? Si ces questions sont souvent posées de manière fugitive ou implicite dans l'enquête historique, l'histoire contrefactuelle les élève au rang d'objets de recherche de plein droit, étudiés à partir de données, d'archives et de preuves d'autres types, et qui peuvent être traités à l'échelle d'articles ou de livres entiers. L'approche contrefactuelle donne cependant lieu à de vives controverses parmi les historiens et dans les autres sciences humaines et sociales, où elle fait aussi l'objet d'un important intérêt, récemment renouvelé, de la part des chercheurs comme des épistémologues ${ }^{1}$. Si ses détracteurs n'y voient souvent qu'un exercice

\footnotetext{
1. Parmi les nombreux exemples récents de cet intérêt chez les historiens français ou étrangers, consulter par exemple : Niall Ferguson (dir.), Virtual History : Alternatives and Counterfactuals, New York, Basic Books, 1999 [1997]; Philip E. Tetlock, Aaron Belkin (dir.), Counterfactual Thought Experiments in World Politics : Logical, Methodological and Psychological Perspectives, Princeton, Princeton University Press, 1996; P. E. Tetlock, Richard N. Lebow et Geoffrey Parker (dir.), Unmaking the West : «What-If?» Scenarios That Rewrite World History, Ann Arbor, University of Michigan Press, 2006; Robert Cowley (dir.), What If? Military Historians Imagine What Might Have Been, Londres, Pan Books, 2001 ; R. Cowley (dir.), More What If? Eminent Historians Imagine What Might Have Been, Londres, Pan Books, 2003; Martin Bunzl, «Counterfactual History : A User's Guide»,
} 
vain et périlleux, ses partisans, au contraire, insistent sur sa dimension empirique et sur sa fécondité. La perspective contrefactuelle, disent-ils, permet de neutraliser les illusions rétrospectives qui guettent l'historien; elle est nécessaire en particulier à qui veut soupeser rigoureusement l'impact causal des faits passés.

Alors que la controverse entre promoteurs et critiques de l'approche contrefactuelle occupe généralement le devant de la scène de l'histoire, la généalogie de cette démarche et l'analyse des clivages méthodologiques ou épistémologiques successifs qu'elle a suscités restent en grande partie à écrire ${ }^{2}$. Présente de manière tacite depuis qu'on écrit de l'histoire, l'approche contrefactuelle est d'abord devenue plus consciente d'ellemême, quoique sous d'autres noms, au XIX ${ }^{\mathrm{e}}$ siècle, où elle existe essentiellement comme forme littéraire («uchronie», « histoire alternative»). On peut aussi considérer que l'on trouve l'une des premières réflexions épistémologiques approfondies sur l'usage des hypothèses «contrefactuelles» en histoire dans les Essais sur la théorie de la science de Max Weber $^{3}$. Alors que cette manière de faire de l'histoire se développe chez certains historiens professionnels dans la première moitié $\mathrm{du} \mathrm{xx}^{\mathrm{e}}$ siècle, en particulier en Grande-Bretagne avec Toynbee par exemple, elle rencontre parallèlement une forte hostilité, plus qu'ailleurs dans ce pays, chez Robin G. Collingwood, Edward H. Carr, puis Edward P. Thompson ou Eric Hobsbawm.

Toutefois, ce n'est que dans les années 1960, avec l'apparition de ce qu'on a appelé la «nouvelle histoire économique» américaine (new economic history), que l'histoire dite «contrefactuelle» est apparue

The American Historical Review, 109/3 (2004), p. 845-858 ; Jacques Sapir, Frank Stora et Loïc Mahé (dir.), 1940. Et si la France avait continué la guerre..., Paris, Tallandier, 2010. Pour les débats sur l'épistémologie des contrefactuels en histoire et sur les discussions que leur usage entraîne, consulter entre autres : Jon Elster, Logic and Society : Contradictions and Possible Worlds, New York, John Wiley, 1978; Geoffrey Hawthorn, Plausible Worlds. Possibility and Understanding in History and the Social Sciences, Cambridge, Cambridge University Press, 1991 ; Julian Reiss, «Counterfactuals Thought Experiments and Singular Causal Analysis in History», Philosophy of Science, 76 (2009), p. 712-723 (dont la traduction par S. Bourgeois-Gironde figure dans le présent numéro).

2. Pour une mise en perspective historique de la pratique contrefactuelle en histoire et des prises de positions qu'elle entraîne, voir cependant Quentin Deluermoz, Pierre Singaravélou, «Explorer le champ des possibles. Approches contrefactuelles et futurs non advenus en histoire», Revue d'histoire moderne et contemporaine, 59/3 (2012), p. 118-143. Voir aussi leur contribution au présent numéro. 3. Max Weber, «Objektive Möglichkeit und adäquate Verursachung in der historischen Kausalbetrachtung», in Gesammelte Aufsätze zur Wissenschaftslehre, Tübingen, Mohr Siebeck, 1988 [1906], p. 266-290. 


\section{Contrefactuels en histoire : du mot au mode d'emploi}

publiquement comme volet important d'un programme collectif de recherches : elle est alors nommée comme telle, elle donne lieu à des travaux d'envergure et suscite un débat de méthode au sein de la communauté historienne - premier d'une série de controverses scientifiques autour de l'usage des contrefactuels ${ }^{4}$. Du fait de l'ampleur que la new economic history a donnée à la pratique des raisonnements contrefactuels, du succès - public comme intellectuel - des recherches conduites sous son étiquette, et du caractère opératoire qu'elle a ainsi su conférer à des manières de faire, certes déjà identifiées, mais encore balbutiantes et peu élaborées, on peut affirmer que les discussions - internes ou externes auxquelles ce courant a pu donner lieu constituent un moment essentiel, quoique encore laissé dans l'ombre, d'une histoire du contrefactualisme au $\mathrm{XX}^{\mathrm{e}}$ siècle. En étudiant attentivement ces discussions, cet article se propose de retracer les lignes de partage méthodologiques et épistémologiques les plus saillantes ayant marqué ce moment d'émergence ${ }^{5}$.

L'histoire intellectuelle des usages du terme «contrefactuel» constitue un point d'entrée privilégié pour une telle enquête. D'après l'Oxford English Dictionary, la première occurrence du terme anglais pour «contrefactuel» date de 1946, sous la forme de l'adjectif «counter-factual», dans un article

\footnotetext{
4. Notons, à titre indicatif, que l'expression complète «histoire contrefactuelle» semble apparaître pour la première fois dans Alexander Gerschenkron, «The Discipline and I», The Journal of Economic History, 27/4 (1967), p. 443-459, où la nouvelle histoire économique américaine fait l'objet d'une analyse. Pour une présentation informée et critique de cette nouvelle histoire économique américaine en langue française quelques années après son émergence comme courant identifié, voir Maurice Lévy-Leboyer, «La "New Economic History" ", Annales. Économies, Sociétés, Civilisations, 24/5 (1969), p. 1035-1069. Les premiers ouvrages de ce courant ayant eu recours de manière centrale au raisonnement contrefactuel, sans que celui-ci soit nécessairement étiqueté de la sorte, sont en particulier : Alfred H. Conrad, John R. Meyer, The Economics of Slavery and Other Studies in Econometric History, Chicago, Aldine, 1964; Robert W. Fogel, Railroads and American Economic Growth: Essays in Econometric History, Baltimore, Johns Hopkins University Press, 1964. Plusieurs articles d'histoire économique ont mis en œuvre des raisonnements contrefactuels avec une modélisation et des données quantitatives dès le milieu des années 1950. Pour l'une de ces premières tentatives, voir en particulier J. R. Meyer, «An Input-Output Approach to Evaluating the Influence of Exports on British Industrial Production in the late 19th Century», Explorations in Entrepreneurial History, VIII (1955), p. 12-34

5. Nous nous concentrons dans notre enquête sur les seules discussions méthodologiques et épistémologiques au sujet de la nouvelle histoire économique américaine et, plus précisément, sur les controverses qui portent explicitement sur l'usage du raisonnement contrefactuel. Les travaux de Robert Fogel, John Meyer, Alfred Conrad, Stanley Engerman, Douglass North et d'autres qui se reconnaissent dans la «nouvelle histoire» sont aussi contestés pour leurs résultats, en particulier, par l'historiographie marxiste, lorsqu'ils portent, comme c'est le cas pour nombre d'entre eux, sur l'histoire de l'esclavage, de la guerre de Sécession ou de la Révolution industrielle.
} 
du philosophe Roderick Chisholm ${ }^{6}$. Le syntagme le plus souvent utilisé par Chisholm est celui de «conditionnel contraire aux faits» (contraryto-fact conditional), expression qui donne son titre à l'article et qui, dans les années qui suivront, le disputera à celles de «counter-factual»ou «counterfactual». Chisholm tient ce syntagme pour sensiblement équivalent à celui de «conditionnel subjonctif» (subjunctive conditional), désignant des énoncés de la forme «si..., alors...» (exprimés en anglais au mode «subjonctif») faisant référence à des possibilités non réalisées, par exemple : «si ce vase tombait par terre, il se casserait», ou «si l'on frottait cette allumette, elle s'enflammerait». Au moment de l'article de Chisholm, ce type d'énoncés préoccupe les logiciens et les philosophes des sciences plutôt que les historiens. Depuis l'article de Ramsey «General Propositions and Causality» (1929) ${ }^{7}$, l'intérêt suscité par ces énoncés tient principalement à ce qu'ils sont sous-entendus à chaque fois que nous exprimons certaines propriétés durables des choses ou les régularités du monde. Les mots «fragile» ou «inflammable», dans les exemples précédents, renvoient ainsi à des propriétés d'un type particulier, qui se manifestent dans certaines conditions, et qu'on appelle «dispositionnelles »; une loi de la nature semble de même impliquer que, dans telle et telle conditions, tel événement se produirait : voilà précisément ce qu'expriment les «subjunctive conditionals». S'ils semblent, dans cette mesure, porteurs d'une vraie connaissance, ils n'en soulèvent pas moins, entre autres difficultés, un problème pour les philosophes : la logique classique est impuissante à rendre compte de leur signification.

En effet, pour la logique traditionnelle, qui définit la valeur de vérité des énoncés composés comme une fonction de celle de leurs composants, tous les conditionnels dont l'antécédent est faux sont trivialement vrais : d'un énoncé exprimant une condition non réalisée, on peut conclure une proposition et son contraire. La logique des fonctions de vérité ne parvient pas à rendre compte de la différence qui existe entre les conditionnels vrais («si ce vase tombait par terre, il se casserait») et les conditionnels faux («si ce vase tombait par terre, il ne se casserait pas») ou absurdes («si ce vase tombait par terre, il deviendrait un chêne»). Chisholm, et d'autres logiciens après lui, ont donc essayé de supprimer ces paradoxes en réduisant les énoncés conditionnels à des conjonctions d'énoncés au

6. Roderick M. Chisholm, «The Contrary-to-Fact Conditional», Mind, n.s., 55/220 (1946), p. 289-307.

7. Frank P. Ramsey, Philosophical Papers, Cambridge, Cambridge University Press, 1990, p. 145-163. 


\section{Contrefactuels en histoire : du mot au mode d'emploi}

mode indicatif. Mais une autre analyse logique, susceptible de rendre compte de leur valeur pour la connaissance, semble nécessaire. Reste à comprendre pourquoi Chisholm appelle «contrefactuels» ces énoncés, qui font référence à des éventualités ou des possibilités réalisables, plutôt qu'à des conditions «contraires aux faits».

Chisholm a choisi ce terme en référence à un autre type de conditionnels : en histoire et dans plusieurs autres sciences, dit-il, il est parfois nécessaire d'envisager les conséquences de conditions impossibles à réaliser et par conséquent de formuler des énoncés conditionnels «contraires aux faits ». Il illustre d'abord cette démarche par un contrefactuel historique, l'hypothèse selon laquelle la Seconde Guerre mondiale ne se serait pas produite si les États-Unis avaient mené une autre politique envers l'Allemagne des années 1920. Autre exemple, puisé dans un autre domaine du savoir : l'expérience de pensée galiléenne dans laquelle, par un passage à la limite, on suppose un solide en mouvement sur lequel ne s'exercerait aucune force extérieure, même si cette situation idéale est concrètement irréalisable. On peut remarquer que cette dernière démarche contrefactuelle ne recouvre pas exactement la démarche hypothétique décrite plus haut. Contrairement aux contrefactuels, les conditionnels subjonctifs qui intéressent au premier chef logiciens et philosophes des sciences ne suppriment pas en pensée un fait réellement advenu ni ne posent un fait impossible; ils font référence à une possibilité qui pourrait advenir à tout moment. Ils n'annulent donc pas la réalité mais la soutiennent et la prolongent par le possible, là où le contrefactuel, surtout historique, invite au contraire à considérer un scénario ou un monde alternatif. Enfin, la réflexion sur le contrefactuel s'inscrit dans des contextes intellectuels très différents dans les deux cas. Le but des philosophes est de parvenir à la clarification logique d'énoncés très ordinaires, alors que l'usage des contrefactuels historiques (ou physiques) est un procédé heuristique plus spécifique supposé nous aider à débrouiller la complexité des enchaînements historiques (ou physiques). Dans le premier cas, le conditionnel est l'objet qu'il faut clarifier; dans le second cas, il est l'outil d'une clarification portant sur le réel. Néanmoins, Chisholm décide d'appliquer le qualificatif «contrefactuel» indifféremment à ces deux types de conditionnels qui ont en commun de poser une hypothèse non réalisée. À l'origine du terme même de «contrefactuel», on trouve donc une hybridation superficielle et partiellement contingente entre une problématique logico-philosophique («comment analyser les conditionnels subjonctifs 
d'une manière logiquement satisfaisante?») et une thématique historienne («que se serait-il passé si X n’avait pas eu lieu?»). Ce lien ténu est cependant immédiatement perdu dans le débat tel qu'il se poursuit chez les philosophes. Un an après l'article de Chisholm, dans un article devenu célèbre intitulé «The Problem of Counterfactual Conditionals ${ }^{8}$ », Nelson Goodman soutient que résoudre le problème des «contrefactuels » - ce dont il s'avoue incapable - reviendrait exactement à donner une analyse satisfaisante des énoncés incluant des prédicats dispositionnels ou formulant des lois de la nature. Goodman reprend à Chisholm le terme de «contrefactuel», tout en le déclarant partiellement inadéquat car le problème logique qu'il a en vue se pose aussi pour d'autres types d'énoncés comme les «conditionnels factuels» («puisque cette allumette ne s'est pas enflammée, c'est qu'elle n'a pas été frottée»). Néanmoins, à partir de l'article de Goodman, c'est bien ce terme (ou celui de conditionnel «contraire-aux-faits») qui servira désormais aux philosophes à désigner spécifiquement ce problème logique9 .

Le mot «contrefactuel» n'a été repris en tant que tel qu'à la fin des années 1950 et dans la décennie suivante par des historiens. Son origine logico-philosophique est bien connue et quelquefois mentionnée dans le débat historiographique portant sur les contrefactuels et le contrefactualisme. Au-delà de cette simple référence, pourtant, ce qui a motivé les historiens à s'intéresser à ces formes d'énoncés, et les modalités selon lesquelles les réflexions philosophiques ont (ou non) alimenté le débat historiographique restent des questions obscures. Nous nous proposons ici de les éclairer en montrant que les premiers usages explicites du terme « contrefactuel» et les discussions qu'ils entraînent en histoire ont connu plusieurs phases bien distinctes après leur apparition en philosophie. Quel est, pour chacune d'entre elles, le contexte d'emploi du mot «contrefactuel»? Une circulation a-t-elle lieu entre ses usages en histoire et ceux qui existent dans d'autres disciplines, en particulier en philosophie, où le mot est né? Quels arguments sont échangés à propos des contrefactuels en histoire ? Comment ces arguments déterminent-ils un épicentre

8. Nelson Goodman, «The Problem of Counterfactual Conditionals», Journal of Philosophy, 44/5 (1947), p. 113-128.

9. Cf. par exemple : Elizabeth L. Beardsley, «"Non-Accidental" and Counterfactual Sentences», Journal of philosophy, 48/18 (1949), p. 573-91; Karl R. Popper, «A note on Natural Laws and so-called "Contrary-to-Fact Conditionals" », Mind, n.s., 58/229 (1949), p. 62-66; Julius R. Weinberg, «Contrary-to-facts conditionals», Journal of philosophy, 48/1 (1951), p. 17-22. 
du débat théorique sur le contrefactualisme en histoire? Telles sont les questions posées de manière récurrente dans cette enquête. Il s'agira ainsi de montrer comment s'est constitué, après l'apparition du terme «contrefactuel» en philosophie, le débat historiographique portant sur le contrefactualisme - tel qu'il se structure dans les années 1960 à propos de la «nouvelle histoire économique» américaine.

Le mouvement général de ce débat se laisse appréhender simplement. La métamorphose en quelques décennies d'après-guerre d'une notion de l'analyse logique en méthode des études historiques s'accompagne parallèlement d'un approfondissement du débat épistémologique sur l'histoire. En transformant un mot en programme de recherches et en discutant ensuite de l'intérêt de ce programme, le discours savant sur les contrefactuels change aussi de ligne de clivage : il n'y est plus seulement question d'accepter ou de rejeter la conception scientifique de l'histoire dont la pratique contrefactuelle serait solidaire, mais de préciser sa pertinence épistémique, son domaine de validité et les stratégies de son usage.

\section{Apparition de la notion chez les historiens : une autonomie relative}

\subsection{Une greffe superficielle}

La première occurrence du terme «contrefactuel» sous la plume d'historiens rattachés à ce que l'historiographie appelle aujourd'hui «new economic history» se trouve chez John R. Meyer et Alfred H. Conrad, dans un article de $1957^{10}$. Bien loin d'être l'objet principal de leur article, la question des «contrefactuels » y est reléguée dans un unique paragraphe qui vient conclure une partie consacrée aux méthodes et données quanti-

10. J. R. Meyer et A. H. Conrad, «Economic Theory, Statistical Inference, and Economic History », The Journal of Economic History, 17/4, (1957), p. 524-544. L'historien de l'économie et sociologue des techniques Seabury Colum Gilfillan s'était déjà interrogé explicitement, dans un article de 1945, sur l'intérêt qu'il y a à formuler en histoire des hypothèses «contraires aux faits» (contrary-tofact) : «Invention as a Factor in Economic History », The Journal of Economic History, 5/S1 (1945), p. 65-85. S'il reconnaît qu'un raisonnement contrefactuel, visant par exemple à déterminer ce qui se serait passé si la machine à vapeur n'avait pas été inventée, serait utile en théorie pour comprendre la contribution de la technique à la Révolution industrielle, il pense qu'il est en pratique impossible à mettre en œuvre. Cependant, son analyse n'est ni reprise ni commentée dans le cadre du débat concernant les contrefactuels tel qu'il se structure dans les années 1950 chez les historiens de l'économie. 
tatives ${ }^{11}$. La thèse défendue est simple : contrairement à ce qu'affirment certains historiens et philosophes de l'histoire, les énoncés qui comportent des conditionnels contrefactuels ou «conditionnels subjonctifs» ne sont pas inaccessibles à toute vérification. La clé est ici la suivante :

Une fois admis que les contrefactuels ne peuvent pas être testés directement, il est possible de les considérer à l'intérieur d'un système déductif valide, indépendamment de la fausseté reconnue de la clause conditionnelle. Ensuite, sans être capable de démontrer l'existence d'une quelconque occurrence du contrefactuel, il peut être possible de vérifier ou de falsifier une autre proposition qui se situe en amont de la chaîne déductive ${ }^{12}$.

Ainsi, poursuivent les deux auteurs, s'il est impossible de tester empiriquement l'énoncé «si la guerre de Sécession n'avait pas eu lieu, le Sud aurait aboli l'esclavage en moins d'une génération», il est possible de tester un énoncé général plus haut placé dans la chaîne hypothéticodéductive, en l'occurrence la proposition selon laquelle «l'esclavage n'était pas économiquement rentable», et la vérité de cet énoncé vient confirmer, ou «soutenir» un équivalent du conditionnel précédemment cité : «même en l'absence de la guerre de Sécession, le système économique de l'esclavage n'était pas rentable, ce qui aurait conduit le Sud à l'abandonner».

Même si, par cet usage du terme «contrefactuel», Meyer et Conrad manifestent certainement quelque chose de l'inventivité dans les méthodes de vérification qui caractérise la new economic history $y^{13}$, ce court paragraphe obéit néanmoins à une logique irréductible aux développements ultérieurs. Pour comprendre exactement ce qui s'y joue, deux éléments de contextualisation sont nécessaires. Le premier relève de l'épistémologie de l'histoire. L'article de Meyer et Conrad prend place dans un projet d'ensemble visant à défendre la possibilité de faire usage, en histoire, d'outils théoriques analogues à ceux des sciences naturelles et de dépasser ainsi, par la théorie et ses généralisations, une conception de l'histoire

11. J. R. Meyer et A. H. Conrad, art. cit., p. 540-541.

12. Ibid., p. 540.

13. Ils résument ainsi leur texte "The Economics of Slavery in the Ante-bellum South», présenté à la Conference on Research in Income and Wealth du National Bureau of Economic Research à Williamstown, Mass. en septembre 1957. 
qui n'y verrait qu'une «collection de faits ou d'énoncés vrais à propos de segments de l'expérience passée ${ }^{14} \gg$. À ce titre, le texte peut être considéré comme une reprise et une réponse à un texte programmatique de Carl G. Hempel datant du début des années 1940, «The Function of General Laws in History ${ }^{15}$ », même si cette continuité relève plus d'une imprégnation que d'une filiation stricte ${ }^{16}$.

Hempel proposait l'application du modèle déductif-nomologique à l'explication historique, faisant ainsi de cette dernière la subsumption de classes d'événements historiques sous des énoncés ayant la forme de lois - une loi étant définie ici comme un «énoncé ayant une forme conditionnelle universelle qui est susceptible d'être confirmé ou infirmé par des découvertes empiriques adéquates ${ }^{17}{ }$. À la suite de Hempel luimême, Meyer et Conrad polémiquent avec les doctrines qui considèrent au contraire l'histoire comme une «description d'événements particuliers ayant eu lieu dans le passé18 ${ }^{18}$, et ils réaffirment la prééminence du modèle explicatif causa ${ }^{19}$. Leur objectif principal est alors de trouver une solution à une difficulté déjà pointée par $\operatorname{Hempel}^{20}$ : la difficulté qu'il y a à formuler, en étant à la fois suffisamment précis, suffisamment général et suffisamment proche des données existantes, les hypothèses à partir desquelles un événement ou une séquence d'événements peuvent être expliqués.

\footnotetext{
14. J. R. Meyer et A. H. Conrad, art. cit., p. 524.

15. Carl G. Hempel, «The Function of General Laws in History», The Journal of Philosophy, 39/2 (1942), p. 35-48.

16. Ce texte apparaît très souvent dans les débats autour de la new economic history et tout particulièrement chez R. W. Fogel (op. cit., p. 1 ou encore «The Specification Problem in Economic History », The Journal of Economic History, 27/3 (1967), p. 289, note 11). Paradoxalement, la filiation textuelle entre ce texte et celui de Meyer et Conrad n'est attestée que par une référence polémique (J. R. Meyer et A. H. Conrad, art. cit., p. 530, note 9). Ce fait s'explique à nos yeux par ceci que le passage de Hempel cité par Meyer et Conrad est (1) un passage où Hempel reconnaît qu'il existe des obstacles à la généralisation en histoire, et (2) un passage tiré d'un livre de Patrick Gardiner (The Nature of Historical Explanation, Oxford, Oxford University Press, 1952) où celui-ci l'utilise à charge contre l'intention initiale de Hempel, à laquelle Meyer et Conrad nous paraissent donc rester fidèles. Meyer et Conrad semblent avoir pris connaissance du modèle hempélien d'explication par l'intermédiaire de Popper et de sa théorie de la falsifiabilité, même s'ils critiquent l'argument poppérien d'après lequel ce modèle ne pourrait s'appliquer à l'histoire (J. R. Meyer et A. H. Conrad, art. cit., p. 529-530).

17. C. G. Hempel, art. cit., p. 35.

18. Ibid.

19. J. R. Meyer et A. H. Conrad, art. cit., p. 525-534.

20. C. G. Hempel, art. cit., p. 40.
} 
Mais là où Hempel semblait en définitive se rétracter et ne reconnaître à l'histoire que la possibilité de mettre en place des «schémas explicatifs » (explanation sketches) inférieurs à de véritables hypothèses explicatives, la démarche de Meyer et Conrad consiste, à l'inverse, à proposer une véritable réponse à cette difficulté. Il s'agit de tirer les conséquences d'une opportunité propre à l'histoire économique qui permet de modéliser mathématiquement les comportements au moyen d'outils statistiques. La discussion portant sur les « conditionnels contrefactuels » intervient dans le cadre d'une réflexion sur ces outils et constitue l'ultime moment d'une série de réponses aux principales objections à une approche quantitative en histoire, notamment celle qui affirme que les hypothèses qu'il s'agirait de tester en histoire seraient plutôt «qualitatives» que quantitatives. La réponse de Conrad et Meyer est qu'il est possible de vérifier certaines hypothèses au moyen de «preuves indirectes» ou de proposer un test quantitatif susceptible de vérifier une hypothèse qualitative à partir des conséquences chiffrées que cette dernière serait susceptible d'avoir si elle était vraie ${ }^{21}$. La réflexion sur les « conditionnels contrefactuels» est introduite comme constituant "l'inverse de ce problème de la déduction indirecte de conséquences ${ }^{22} \gg$ : là où il s'agissait de partir d'une hypothèse comme si elle était vraie (et elle pourrait bien l'être, en effet) pour comparer les données déduites et les données historiquement disponibles, il s'agit dans le cas des énoncés au « conditionnel contrefactuel» de partir d'un énoncé dont on sait qu'il est faux pour chercher à en déduire des conséquences. Comme nous l'avons vu, Meyer et Conrad ne se laissent pas plus arrêter par cette difficulté que par les précédentes : ils proposent de s'appuyer sur les relations logiques (de confirmation en particulier) que le «conditionnel contrefactuel» entretient avec des énoncés testables appartenant au même système déductif.

Alors que les objections à la quantification paraissaient endogènes à la pratique de l'histoire, et plus particulièrement de l'histoire économique, l'apparition dans ce cadre de la référence philosophique aux «conditionnels contrefactuels » peut paraître surprenante. Pour prendre la pleine mesure de ce qu'elle signifie, il convient de revenir sur un second élément de contexte, identifiable dans les notes de Meyer et Conrad : les «conditionnels contrefactuels» ne sont évoqués qu'au titre

21. J. R. Meyer et A. H. Conrad, art. cit., p. 538-539.

22. Ibid., p. 540. Souligné par nous. 
d'un certain état du débat philosophique à leur propos, en l'occurrence avec la signification que leur avait donnée le philosophe anglais Stuart Hampshire (1914-2004) dans un article publié en 1948, après ceux de Chisholm et de Goodman que nous avons mentionnés plus haut ${ }^{23}$. Hampshire considère les propositions contrefactuelles à la fois comme des énoncés indispensables à l'historien, voire symptomatiques du travail spécifique de l'histoire - consistant à évaluer les responsabilités causales et à interpréter les séquences causales -, et comme des énoncés dont la forme logique pose des difficultés telles qu'elles interdisent purement et simplement de considérer l'histoire comme une «science» au sens hypothético-déductif du terme :

On attend professionnellement de l'historien, écrit Hampshire, qu'il produise des jugements de la forme «Si Hitler avait envahi la GrandeBretagne en 1940, il aurait conquis Londres». S'il était mis au défi de traduire ce jugement en un ensemble d'énoncés falsifiables en termes de logistique (au sens militaire), il en serait incapable et ne le voudrait pas $^{24}$.

En somme, les énoncés impliquant des « conditionnels contrefactuels » sont considérés par Meyer et Conrad - qui suivent sur ce point Hampshire sans plus entrer dans le détail du débat philosophique - comme la pierre de touche de tout rattachement de l'histoire à un modèle hypothéticodéductif de scientificité, autrement dit comme l'ultime difficulté logique qu'aurait à affronter une définition de l'histoire comme explication ${ }^{25}$. Puisque, pour Hampshire, ces énoncés et les difficultés qu'ils posent rendent nécessaire de distinguer radicalement entre la science - qui fonctionne par test d'hypothèses - et l'histoire - qui fonctionne par justification de jugements -, le fait de pouvoir proposer, pour ces énoncés, un analogue vérifiable, fût-ce indirectement, constitue indéniablement le point d'orgue de la démarche de Meyer et Conrad. Le bref paragraphe consacré aux «conditionnels contrefactuels» est alors à la fois un tour de force permettant de mettre en évidence la puissance de leur approche

23. Stuart Hampshire, «Subjunctive Conditionals», Analysis, 9/1 (1948), p. 9-14. Notons que S. Hampshire, même s'il a enseigné à Princeton entre 1963 et 1970, est anglais et que son article a été publié à Oxford, et non aux États-Unis.

24. Ibid., p. 12.

25. J. R. Meyer et A. H. Conrad, art. cit., note 2. 
quantitative, une démonstration de leur inventivité méthodologique, et une preuve a fortiori de la validité du modèle hypothético-déductif en histoire. D'une certaine manière, Meyer et Conrad ouvrent donc bien la voie à une assimilation des énoncés comportant des «conditionnels contrefactuels » à des hypothèses testables et donc à leur intégration à part entière dans une histoire scientifique.

\subsection{Un terme de référence plutôt qu'une théorie de la pratique savante}

Il faut néanmoins souligner que, si Meyer et Conrad, d'une part, et Robert Fogel, de l'autre, renvoient bien sous le qualificatif «counterfactual » au même type d'énoncés, la notion de contrefactuel telle qu'elle fait son apparition chez les premiers est très différente de celle qui est utilisée plus tard par le second. La première différence porte sur la signification des énoncés contrefactuels pour l'histoire. Meyer et Conrad s’intéressent à ces énoncés exclusivement par l'intermédiaire d'une problématique philosophique : ces énoncés ne sont pas considérés pour eux-mêmes, en tant qu'ils pourraient être produits ou formulés par les historiens, mais à travers leur signification dans le débat sur la scientificité de l'histoire ${ }^{26}$. Si l'article de Meyer et Conrad n'est pas une contribution au débat philosophique et logique sur les énoncés contrefactuels ${ }^{27}$, il ne s'agit pas non plus d'une importation de ces débats dans le champ de l'histoire économique. Or, pour Fogel, les énoncés contrefactuels font au contraire sens immédiatement dans le cadre d'une pratique de recherche et de test des assertions causales, plutôt que dans une optique qui serait d'abord philosophique.

Il faut ensuite noter qu'aucune préconisation méthodologique à raisonner contrefactuellement n'accompagne la démonstration par Meyer et Conrad de la possibilité de tester des énoncés impliquant des «conditionnels contrefactuels ». Par contraste, pour Fogel, ces énoncés sont amenés à apparaître dans toute argumentation visant à établir l'importance d'un

\footnotetext{
26. Si une seconde référence philosophique à l'article de Goodman de 1947 vient clore le développement sur les énoncés contrefactuels, elle nous paraît peu significative dans la mesure où l'argumentation de ce dernier n'est ni intégrée, ni même développée - contrairement à celle de Hampshire.

27. J. R. Meyer et A. H. Conrad, art. cit., p. 540.
} 
facteur causal et, plus généralement, dans toute analyse comportant une dimension comparative ${ }^{28}$.

Il convient enfin de pointer les nuances épistémiques dont Meyer et Conrad entourent leur méthodologie, lesquelles disparaissent dans l'usage des contrefactuels par Fogel. Ce qui est testable pour les deux historiens de Harvard, en effet, ce ne sont pas les propositions contrefactuelles elles-mêmes, mais « des propositions en amont dans la chaîne déductive». Cela introduit bien entendu une limite à la testabilité des énoncés contrefactuels : si l'on peut considérer que la loi comportementale générale «si un mode de production - par exemple l'esclavage - n'est pas rentable, les agents économiques tendent à en changer» confirme (ou soutient) l'hypothèse «si la guerre de Sécession n'avait pas eu lieu, le Sud aurait fini par abolir l'esclavage», elle n'établit pas, à proprement parler, sa vérité. Il serait notamment possible de formuler d'autres énoncés généraux capables de «couvrir» et d'expliquer une abolition de l'esclavage en l'absence de guerre de Sécession. Ainsi le premier usage du terme philosophique de «contrefactuel» chez les historiens ne renvoie-t-il ni à une démarche méthodologique d'ensemble ni à un mode de raisonnement incontestable. Il est mobilisé avant toute chose afin de défendre une conception déductive-nomologique de l'explication historique et un ensemble d'instruments au service de ce but. C'est seulement dans les années 1960 avec les recherches de Robert Fogel que le terme de «contrefactuel» acquiert chez les historiens une ampleur programmatique et une signification autonome vis-à-vis de la philosophie.

28. Y compris la comparaison entre événements réels. Cf. R. W. Fogel, «The New Economic History : Its Findings and Methods», Economic History Review, 19/3 (1966), p. 642-656, ici p. 655. 


\section{Cristallisation et polarisation : controverses autour des travaux de Robert Fogel}

\subsection{Les contrefactuels chez Robert Fogel et la nouvelle histoire économique}

La pratique fogelienne du raisonnement contrefactuel est attestée dans ses travaux des années $1960^{29}$. Elle consiste à évaluer l'importance d'un facteur causal en construisant un modèle permettant de savoir quelle direction aurait prise la réalité si ce facteur causal n'avait pas existé, et dont le ressort est la comparaison entre les données chiffrées factuelles concernant la réalité impactée et les données chiffrées contrefactuelles ${ }^{30}$. Mais le terme «contrefactuel» n'y apparaît pas alors, ni pour désigner cette pratique, ni pour faire référence aux débats philosophiques sur la question. La pratique fogelienne est donc d'abord baptisée «contrefactuelle» par ses détracteurs, même si Fogel reprend ensuite le terme sans le contester. Ce n'est en effet que dans un article de 1966 présentant les méthodes et résultats de la new economic history que le terme «contrefactuel» est utilisé sous la plume de Fogel pour la première fois afin de décrire une manière de faire de l'histoire :

L'effet net [des innovations, des institutions ou des processus sur le cours du développement économique] [...] engage une comparaison entre ce qui est effectivement arrivé et ce qui serait arrivé en l'absence de la circonstance spécifiée. Toutefois, puisque la condition contrefactuelle n'a jamais eu lieu, elle n'a pas pu être observée, si bien qu'elle n'est pas enregistrée dans des documents historiques. Afin de déterminer ce qui serait arrivé en l'absence d'une circonstance donnée, l'historien économique a besoin d'un ensemble de généralisations (c'est-à-dire un ensemble de théories ou un modèle) qui lui permettront

29. R. W. Fogel, «A Quantitative Approach to the Study of Railroads in American Economic Growth : A Report of Some Preliminary Findings», Journal of Economic History, 22/2 (1962), p. 163-197 et Railroads and American Economic Growth, op. cit.

30. En cas de recoupement, l'importance causale est quasi nulle; en cas de divergence notable, on peut considérer qu'il s'agissait d'un facteur décisif. Cf. R. W. Fogel, «The New Economic History», art. cit., p. 653 . 


\section{Contrefactuels en histoire : du mot au mode d'emploi}

de déduire une situation contrefactuelle des institutions et des relations qui ont effectivement existé ${ }^{31}$.

Et c'est aussi dans le cadre d'une présentation des résultats et des méthodes de la «nouvelle histoire économique» qu'un autre historien présente la construction de réalités «contrefactuelles» comme l'un des quatre traits principaux de ce programme de recherches :

Même si le terme n'a pas été défini précisément, il apparaît que la «nouvelle» histoire essaie de faire quatre choses. D'abord, établir précisément les questions soumises à l'examen et définir de manière opérationnelle quelles sont les variables pertinentes. Deuxièmement, construire des modèles explicites qui sont pertinents pour la question qui est en jeu. Troisièmement, produire des données (souvent quantitatives mais parfois qualitatives) à propos du monde tel qu'il a réellement existé. Et enfin, tester le modèle (un énoncé logique d'hypothèses et de conclusions) en regard des données (le monde qui a existé) et de sa déduction contrefactuelle (le monde qui n'a pas existé). [...] C'est seulement à travers une comparaison entre ce qui a été et ce qui aurait pu être que nous sommes en mesure de produire des énoncés sur la nature des événements ${ }^{32}$.

Le terme «contrefactuel» était certes déjà présent chez Meyer et Conrad, mais ce sont donc les travaux de Fogel et les discussions qu'ils entraînent qui constituent le principal point d'inflexion à la fois dans la sémantisation du terme, dans son appropriation par les historiens et dans la constitution des arguments concernant les énoncés contrefactuels. Avec la pratique fogelienne du raisonnement contrefactuel, qui va devenir le symbole de la new economic history, le statut épistémologique des énoncés au «conditionnel contrefactuel » a changé : de cas limite et problématiques d'une application possible d'un modèle «scientifique», hypothéticodéductif, à la connaissance historique, ils deviennent parfaitement solubles

31. Ibid.

32. Lance Davis, «The New Economic History. II. Professor Fogel and the New Economic History», The Economic History Review, n.s., 19/3 (1966), p. 657-663, ici p. 657-658. 
dans un tel modèle ${ }^{33}$. Plus encore, ce sont des conséquences inévitables de toute explication causale.

La définition de la nouvelle histoire économique à partir des démarches contrefactuelles particulièrement saisissantes auxquelles elle a donné lieu dans les travaux de Fogel et la focalisation sur ces démarches pour distribuer les positions concernant les contrefactuels proviennent de l'intérieur même du débat et semblent être le fait de certains de ses protagonistes - au premier rang desquels Fogel lui-même, dans les polémiques successives qui l'ont opposées à Fritz Redlich et Robert L. Basmann. On peut considérer que cette polarisation est définitivement installée à partir de $1967^{34}$. Pourtant, nous voudrions montrer que cette période ne constitue elle-même qu'une phase terminale dont la genèse mérite d'être retracée. Lorsque l'on considère le débat autour de la new economic history depuis la perspective qui sera celle de Fogel à partir de 1966, Fritz Redlich, avec son article «"New" and Traditional Approaches to Economic History and Their Interdependence ${ }^{35} »$, apparaît, à tort ou à raison, comme l'adversaire principal de la pratique contrefactuelle en histoire et, de ce fait, de la new economic history. Et c'est la manière dont Fogel reçoit les critiques de Redlich qui contribue à fixer une bonne partie des argumentaires pro et contra.

\subsection{La critique des nouveaux historiens économiques par Fritz Redlich (1965)}

La critique de Redlich envers Meyer, Conrad et Fogel s'appuie sur une notion forgée par l'historien allemand émigré, celle de «figment». Ce terme est tout d'abord introduit par Redlich comme un équivalent

\footnotetext{
33. R. W. Fogel, «The New Economic History», art. cit., p. 655 : «les propositions contrefactuelles sont simplement des inférences à partir de modèles hypothético-déductifs».

34. Outre la position de Fogel, qui tend significativement à faire de son propre usage des raisonnements contrefactuels le coeur du débat sur la new economic history, on peut également évoquer A. Gerschenkron (art. cit.) qui met lui aussi cette question de l'utilisation des raisonnements contrefactuels au centre de sa reconstruction des débats. Deux ans plus tard, J. D. Gould se contentera également, dans le regard rétrospectif qu'il jette sur les débats des historiens de la new economic history, de considérer ce problème méthodologique comme l'enjeu originaire du débat: «Hypothetical History», The Economic History Review, n.s., 22/2 (1969), p. 195-207.

35. Fritz Redlich, «"New" and Traditional Approaches to Economic History and Their Interdependence», The Journal of Economic History, 25/4 (1965), p. 480-495.
} 


\section{Contrefactuels en histoire : du mot au mode d'emploi}

du terme allemand «Fiktion»- «figment» étant ici préféré au terme anglais «fiction»- qui avait été utilisé par Hans Vaihinger pour désigner des «constructions mentales» «sur le mode du "comme si" ${ }^{36}$ ». Il s'agit pour Redlich de remettre en question une opposition basique qu'il attribue, non sans reproche, à la plupart des historiens. Ces derniers admettraient que l'on formule en histoire des hypothèses vérifiables mais rejetteraient purement et simplement tout usage de «figments», sous prétexte que le recours à un élément fictionnel conduirait nécessairement à une histoire conjecturelle. Contre cette dichotomie simplifiée, Redlich entend requalifier certains usages de «figments» en montrant que leur caractère fictionnel ne les empêche pas nécessairement d'avoir une valeur de connaissance. Il propose donc de distinguer hypothèses et figments à partir de trois critères.

Le premier, le rapport à la réalité empirique, permet d'opposer l'hypothèse, qui a une «contrepartie réelle», voire constitue un «reflet» de la réalité et en est dérivée, et le «figment», qui n'a pas de contrepartie réelle ou bien, s'il en a une, a une contrepartie «irréaliste ${ }^{37} »$ et constitue donc une pure «construction sur le mode du "comme si" ". Le deuxième critère de différenciation entre hypothèse et «figment» conduit à les opposer en fonction de leur mode de validation : tandis qu'une hypothèse peut être vérifiée ou falsifiée, un «figment» ne peut pas l'être, mais il peut être justifié par son utilité. Cette dernière détermination peut être précisée à partir du troisième critère proposé par Redlich - le résultat de leur emploi en termes de connaissance : si l'hypothèse, une fois vérifiée ou falsifiée, produit bien une connaissance de la réalité, un «figment» bien justifié constitue quant à lui «un outil pour l'acquisition de connaissance».

En proposant une distinction entre hypothèse et «figment», Redlich peut certes récuser, au nom de l'impératif de vérification et du modèle de connaissance hypothético-déductif, toute connaissance fondée sur des «figments » dès lors qu'elle se prétend vérifiable. Mais il peut également déterminer une sphère dans laquelle la construction de «figments»a une légitimité, sphère qui n'aura pas le même but de connaissance que l'histoire - qui reste la description de la réalité empirique - mais aura

\footnotetext{
36. Ibid., p. 484.
}

37. Même si Redlich ne donne pas d'exemples de «figments» ayant une contrepartie «irréaliste», on voit bien comment les raisonnements contrefactuels consistant à raisonner à partir d'une condition dont on sait qu'elle est fausse peuvent trouver ici leur place. 
pour fin la construction de modèles. Une deuxième ligne d'argumentation se dessine alors : plutôt que de contester les «figments» au nom de leur invérifiabilité, il devient possible de plaider l'irréductibilité de deux pratiques tout aussi légitimes l'une que l'autre, mais dont l'une, seule, devra être appelée «histoire». C'est la voie choisie par Redlich, qui reconnaît la valeur et la puissance cognitive des différents types de «figments» utilisés par Fogel ou Meyer et Conrad en tant que « constructeurs de modèles ${ }^{38}$ », tout en contestant que leur démarche puisse être considérée comme de l'histoire ${ }^{39}$. L'argument de Redlich contre le groupe des nouveaux historiens de l'économie concerne donc la confusion entre ces deux sphères et le risque qu'il y a à substituer un modèle à la réalité historique, «car c'est bien d'un outil qu'il s'agit et pas d'un bien de consommation».

Reste à déterminer quel rapport spécifique cette ligne argumentative entretient avec la question des contrefactuels. La seule et unique occurrence du terme «counterfactual» dans l'article de Redlich pose apparemment une pure et simple identité entre «figments » et contrefactuels - et c'est d'ailleurs de cette façon que Redlich interprétera le travail de Fogel :

Sans aucun doute, ce que, dans la droite ligne de la tradition philosophique allemande, $\mathrm{j}$ 'ai appelé «figment» a récemment fait son apparition en Amérique sous le nom de «conditionnel contrefactuel, ou au conditionnel ${ }^{40} »$.

Mais de quelle identité s'agit-il ici ? Certainement pas d'une identification du concept de «figment» avec ce que Fogel appellera ensuite «contrefactuel», - sans plus de référence au débat philosophique, et sans guillemets - à savoir sa propre pratique ${ }^{41}$. Certes, les raisonnements contrefactuels, tels que Fogel les pratique et les conçoit, ont leur place dans

\footnotetext{
38. Ibid., p. 490.

39. «Un modèle n'est jamais de l'histoire, car il est conjecturel ou au conditionnel ou, dans les termes que Max Weber utilise pour parler des idéaux-types, une distorsion de la réalité»,loc. cit.

40. Ibid., p. 484-485. Redlich cite à ce propos l'article de Stuart Hampshire déjà évoqué par Meyer et Conrad: S. Hampshire, art. cit.

41. Même si la déformation imposée par Fogel aux positions de Redlich doit à notre sens être comprise avant tout comme stratégique, on conçoit également qu'une telle identification explicite, couplée aux attaques ad hominem dont il fait l'objet de la part de Redlich, ait pu suggérer à Fogel que par «figment » il fallait entendre «contrefactuel» et par «contrefactuel» sa propre démarche. À ce titre, il est même envisageable de voir dans cet énoncé l'une des raisons pour lesquelles Fogel s'est mis aussitôt après la parution de l'article de Redlich à revendiquer pour sa propre pratique le terme «counterfactual».
} 
le concept de «figment», au moins à deux titres : en raison du caractère «figmentaire» de la démarche consistant à supprimer un facteur qui a existé historiquement et en raison du caractère «figmentaire» du modèle employé pour produire des inférences à partir de cette suppression et des données réelles. Mais cela ne signifie pas que les raisonnements contrefactuels épuisent le contenu du concept de «figment». La démarche contrefactuelle de Fogel est traitée par Redlich comme un cas particulier de modélisation et non comme une pratique spécifique ou comme le cœur du problème posé par la new economic history. De fait, la critique d'ensemble par Redlich du groupe des «constructeurs de modèles » porte plutôt sur le bon usage de la modélisation et de la théorie, comme le révèle la conclusion de son article ${ }^{42}$, et c'est à ce titre qu'elle enveloppe également la question des contrefactuels. En affirmant une identité entre figments et «conditionnels contrefactuels», Redlich entend en fait proposer une traduction d'un dispositif épistémologique importé (le néo-kantisme de la tradition Herbarth-Lotze-Vaihinger probablement rapporté de sa formation en Allemagne) dans les termes du débat philosophique sur les « conditionnels contrefactuels », lui aussi exogène à l'histoire économique mais que Redlich présuppose sans doute mieux connu de ses lecteurs américains. Il contribue en tout cas à installer le terme dans l'horizon du débat d'histoire économique, en entérinant la référence au terme et à un certain corpus.

Cependant, si l'on se penche sur la possible signification épistémologique de cette identification, il semble que ce rapprochement constitue, comme chez Meyer et Conrad, une reconnaissance du sens que Hampshire donne aux «conditionnels contrefactuels » plutôt qu'une prise en compte de leur spécificité en histoire économique. Deux éléments du texte de Hampshire nous paraissent expliquer le rapprochement effectué par Redlich. Le premier est l'opposition entre «vérification» et «justification», que l'on retrouve à la racine de sa distinction entre «hypothèse » et «figment» et qui semble trouver sa source dans la mise au jour par Hampshire de deux types d'énoncés comportant des «conditionnels contrefactuels» : ceux qui sont susceptibles d'être remplacés par la combinaison d'un énoncé général et d'énoncés particuliers décrivant les conditions initiales auxquelles il s'applique ${ }^{43}$, et qui sont donc susceptibles

42. F. Redlich, art. cit., p. 494-495.

43. S. Hampshire, art. cit., p. 10. 
d'être vérifiés; et ceux dans lesquels le «conditionnel au conditionnel» est inéliminable parce qu'il exprime un jugement ou une interprétation, qui peut être justifiée mais pas vérifiée ${ }^{44}$. Le second élément est le fait que les «conditionnels contrefactuels» exprimés au conditionnel ont, pour Hampshire, quelque chose de trompeur : s'ils semblent toujours envelopper un énoncé général et, par conséquent, une vérification ou une falsification possible ${ }^{45}$, ils peuvent dans certains cas dissimuler un jugement ou une interprétation. Cette thèse a pu attirer l'attention de Redlich, qui entendait dénoncer comme une illusion la prétention fogelienne à proposer de véritables vérifications de ses énoncés contrefactuels.

\subsection{Réponses de Fogel et leçons de la controverse}

Fogel résume la position de Redlich à partir de deux thèses qu'il lui attribue et auxquelles il répond par des arguments symétriques. La première thèse est que les «propositions contrefactuelles» ne seraient pas susceptibles d'être vérifiées parce que, nous l'avons vu, elles seraient en réalité non pas des hypothèses, mais des «figments», qui ne permettent de faire que de la «quasi-histoire ${ }^{46} »$. La réponse de Fogel consiste à récuser cette distinction et à exhiber trois moyens de vérifier de telles assertions - qui ne sont, selon lui, «rien d'autre que des inférences à partir d'un modèle hypothético-déductif ${ }^{47} \gg-$ trois moyens qui sont aussi les moyens classiques de vérification dans un modèle hypothéticodéductif : démontrer la cohérence des inférences en question, montrer la validité empirique du modèle et vérifier son exactitude au moyen de prédictions. La seconde thèse attribuée par Fogel à Redlich est que le questionnement contrefactuel ferait le partage entre old et new economic history. La riposte de Fogel est alors la suivante : répondre aux questions contrefactuelles est inséparable de l'explication historique comme telle et constitue la seule et unique justification des assertions causales au sujet de l'importance de tel ou tel facteur; la new economic history ne ferait que spécifier ce que les historiens «classiques» laissaient dans

44. Ibid., p. 12.

45. Ibid., p. 10.

46. R. W. Fogel, «The New Economic History», art. cit., p. 643, 655.

47. Ibid., p. 655 . 
l'ombre, à savoir les modèles d'explication sous-jacents à telles ou telles assertions causales ${ }^{48}$.

Sans être inexacte, cette reconstitution par Fogel de l'argumentaire de Redlich pose néanmoins problème. Attribuer à ce dernier la thèse selon laquelle ce serait l'usage des raisonnements contrefactuels qui ferait le partage entre histoire économique classique et nouvelle histoire économique constitue une interprétation forcée. L'article de Redlich, comme l'attestent son titre et les développements de sa dernière partie ${ }^{49}$, entend montrer l'«interdépendance» entre approches nouvelles et anciennes. En outre, Redlich conteste qu'il y ait même une new economic history unifiée. Après avoir écarté tous les critères par lesquels la new economic history a cherché à se définir, notamment chez Fogel (par le type d'outils mathématiques utilisés, par un projet tourné vers la mesure et la quantification, etc.) et montré qu'ils n'ont rien de proprement nouveau, le seul et unique critère subsistant pour Redlich - «l'usage d'hypothèses ou de figments ${ }^{50} \gg-$ est un critère composite, qui ne fait pas tant le partage entre new et old economic history qu'entre plusieurs mouvements au sein de la première, qui se rapportent très différemment à la tradition de l'histoire économique ${ }^{51}$. Redlich identifie certes un groupe particulièrement «nouveau » et problématique, celui que forment Fogel, Meyer et Conrad et dont la spécificité est de proposer une histoire «fondée sur des figments», qui n'est alors plus de l'histoire mais de la « quasi-histoire» ou de la «construction de modèles». Mais il ne va pas de soi pour Redlich que l'unité de ce groupe soit à trouver dans la pratique de raisonnements contrefactuels à la Fogel. Dans la mesure où l'article de Redlich n'est pas focalisé sur les raisonnements contrefactuels, cette focalisation semble bien, dans une très large mesure, être due à Fogel lui-même. Il reste que sur le plan de l'évolution sémantique du terme «countrefactuel», l'article de Redlich et les réponses qu'il a entraînées chez Fogel ont bel et bien contribué - en venant redoubler la première importation du mot par Meyer et Conrad - à imposer le terme, en identifiant la question du «figment», centrale dans l'analyse de Redlich, et sa critique de la new economic history, à celle des «conditionnels contrefactuels».

48. Ibid., p. 655-656.

49. F. Redlich, art. cit., p. 491-495.

50. Ibid., p. 485.

51. Ibid., p. 491. 
Sur le plan épistémologique, l'intervention de Redlich vient par ailleurs confirmer le terrain sur lequel le débat se déroule : celui d'une application du modèle hypothético-déductif à la connaissance historique, qui constituait déjà le but visé par Meyer et Conrad et qui continuera à être l'enjeu des réflexions de Fogel ${ }^{52}$. À la suite de ce moment paroxystique qui semble culminer dans les années 1965-1967 avec la discussion entre Robert Fogel et Fritz Redlich, s'ouvre une troisième phase du débat historiographique, dans laquelle la distribution des positions sur les contrefactuels en histoire se complexifie et s'enrichit : en lieu et place d'un débat polarisé par la seule question de savoir si l'on est pour ou contre l'usage des contrefactuels - et ipso facto pour ou contre la new economic history - il s'agit désormais de préciser et de détailler différents types de raisonnements et de pratiques contrefactuels ainsi que de déterminer les limites qu'il convient de poser à leur usage.

\section{Modes d'emploi du contrefactualisme en histoire économique}

\subsection{Pour un contrefactualisme limité}

L'entrée dans cette troisième phase de discussion entre historiens et théoriciens de l'histoire sur l'usage des contrefactuels est marquée, dans les revues savantes, par deux contributions qui reviennent sur les travaux de Fogel et de la new economic history.

La première est issue du discours prononcé par Alexander Gerschenkron, alors professeur à Harvard et président de l'Economic History Association, lors du congrès de celle-ci. Avec toute la signification institutionnelle que cette prise de position confère à son geste, il reconnaît alors l'importance de la new economic history, la validité de ses méthodes et de ses outils - notamment les pratiques contrefactuelles - et commence à orienter le débat vers des distinctions nouvelles ${ }^{53}$. Il est aussi le premier des protagonistes des controverses étudiées ici à employer l'expression d'«histoire contrefactuelle», définie comme «histoire au mode conditionnel ${ }^{54} »$.

52. «La spécificité méthodologique fondamentale de la new economic history est sa tentative de couler toutes les explications du développement économique passé dans le moule de modèles hypothéticodéductifs valides» (R. W. Fogel «The New Economic History», art. cit., p. 656).

53. A. Gerschenkron, «The Discipline and I», art. cit.

54. Ibid., p. 454. 
Si son discours inaugural porte en partie sur le nouveau courant de la discipline, c'est que celui-ci a rencontré une forte hostilité qui menace de diviser la profession. Gerschenkron entend, quant à lui, apaiser les esprits et il en appelle à des discussions scientifiques recentrées sur les études de cas et non plus exclusivement sur les questions de méthode.

À l'inverse des épistémologues de l'histoire qui voudraient exclure le recours aux modèles de cette discipline, quitte à la situer hors de toute scientificité, il affirme tout d'abord que toute démarche scientifique «procède par abstraction et généralisation ${ }^{55}$ ». Ainsi, l'histoire économique, si elle «traite toujours des motivations humaines», peut le faire «d'une manière parfois plus abstraite et généralisatrice, parfois moins », si bien qu'il existe «une variété infinie dans le degré d'abstraction avec lequel nous pouvons désirer approcher l'étude de notre sujet ${ }^{56} »$. C'est donc la qualité de la contribution apportée à la connaissance et l'adéquation entre l'objectif scientifique visé et les méthodes choisies qui doit primer et il ne saurait dès lors être question, conclut-il, de disqualifier certaines manières de faire de l'histoire économique, en particulier la «nouvelle histoire économique», contestée parce qu'elle pratique la modélisation et recourt à des données quantitatives ${ }^{57}$.

La dernière partie du discours de Gerschenkron, la plus longue, est alors consacrée à un «problème méthodologique» soulevé par des «études récentes en histoire économique», qui entendent «évaluer la signification quantitative d'un événement économique donné de manière différentielle, c'est-à-dire en le comparant avec une situation qui aurait été obtenue en l'absence de cet événement ${ }^{58} \gg$. Or, tandis que, selon Gerschenkron, les partisans de l'histoire contrefactuelle voient là la seule manière correcte de faire de l'histoire économique, voire affirment que toute relation de causalité est contrefactuelle par essence, leurs adversaires rejettent purement et simplement en dehors de la discipline, à la manière d'E.H. Carr ou de Robin G. Collingwood, l'histoire de ce qui aurait pu être. Cette opposition lui paraissant trop frontale et idéologique, l'Autrichien émigré aux États-Unis voudrait tracer une voie tierce en proposant des critères d'usage des contrefactuels.

55. Ibid., p. 446.

56. Ibid.

57. Ibid., p. 447.

58. Ibid., p. 454. 
Outre l'attrait pour l'esprit et la curiosité qu'ont à ses yeux les raisonnements contrefactuels, il reconnaît, d'une part, que des énoncés factuels admis peuvent cacher en leur sein des «opérations contrefactuelles» et que, d'autre part, le raisonnement comparatif, lui aussi, s'appuie parfois sur de telles opérations ${ }^{59}$. Gerschenkron poursuit cependant en récusant l'idée de Fogel selon laquelle l'histoire économique ne pourrait pas se passer de raisonnements contrefactuels parce que ces derniers représenteraient un outil inestimable pour établir des relations causales robustes et faire de l'histoire une véritable science. La charge du président de l'Association d'histoire économique porte précisément sur l'idée souvent défendue par Fogel, en particulier dans sa réponse à Redlich, selon laquelle une proposition contrefactuelle se cache derrière tout énoncé de relations causales.

Les relations causales, dit Gerschenkron, sont forgées à partir de faits ou de leur contraire mais pas nécessairement à partir de contrefactuels. «Ce qui est vrai c'est que n'importe quel énoncé factuel affirmatif, qu'il soit causal ou d'une autre nature, implique le rejet de sa négation ${ }^{60} »$. Il est par conséquent possible de réfuter un grand nombre de relations causales existant dans la réalité à partir d'une enquête, en établissant des faits contraires, mais sans recourir ainsi pour autant à des contrefactuels. Pour le professeur d'Harvard, le raisonnement contrefactuel n'est donc pas applicable universellement à toute recherche de causalité. Il suppose, d'une part, un questionnement causal particulier (établir une responsabilité plutôt que simplement établir les causes) et il doit, d'autre part, être réservé à des problèmes spécifiques.

Les problèmes pertinents pour l'analyse contrefactuelle concernent premièrement «l'élucidation de changements de relativement court terme, de préférence lorsque le facteur politique peut être en grande partie négligé». La raison d'une telle restriction est bien simple : «une fois que la période étudiée s'allonge, le nombre de facteurs inconsidérés et non envisageables qui pèsent sur la conséquence augmente rapidement et la signification des résultats diminue encore plus vite ${ }^{61} »$. Une deuxième condition d'usage des contrefactuels devrait viser à en subordonner le recours à une recherche factuelle préalable la plus exhaustive possible,

59. Ibid., p. 455.

60. Ibid., p. 456.

61. Ibid., p. 457 pour les deux citations. 
visant, dans le meilleur des cas, à explorer toutes les relations envisageables entre événements en fonction des données disponibles ${ }^{62}$.

En érigeant ces deux critères d'usage des contrefactuels en histoire économique, Gerschenkron se démarque nettement de la condamnation des contrefactuels comme fictions par les historiens traditionnels de l'économie et par une grande partie des historiens de la période. Mais il rejette tout autant la défense inconsidérée de ce type de raisonnement au sein de la new economic history et de ses partisans, a fortiori lorsque celle-ci s'appuie sur une épistémologie implicite qui, comme celle de Fogel, entend faire de l'histoire une science nomologique. L'histoire n'a pas de lois, dit l'historien de Harvard ailleurs dans cette allocution, et s'il est légitime, en tant que savant, de rechercher des hypothèses générales, tout chercheur responsable doit également en établir les limites ${ }^{63}$.

\subsection{Situer la validité de l'approche contrefactuelle}

On trouve une orientation générale comparable dans l'article d'un historien de l'économie britannique à l'époque moderne, John Dennis Gould, paru en 1969 dans l'Economic History Review, où Gerschenkron avait publié son allocution. Gould est le premier contributeur de la controverse autour de la nouvelle histoire économique qui déplore l'absence de liaison entre cette polémique et le débat philosophique des années 1940 autour des contrefactuels ${ }^{64}$. Il propose une critique non pas seulement empirique ou épistémologique mais logique et conceptuelle de l'emploi des contrefactuels dans la nouvelle histoire économique. Car, dit-il, «l'historien au travail, en essayant d'estimer la valeur de l'argument contrefactuel dans sa discipline, se retrouve rapidement face à des difficultés qui semblent être des manifestations de deux problèmes majeurs liés aux contrefactuels, problèmes sur lesquels l'analyse philosophique s'est concentrée», en particulier Goodman, dont l'article de 1947 est mentionné ${ }^{65}$. «Ces problèmes, poursuit-il, concernent (i) le type de généralisations capable de soutenir des contrefactuels et (ii) l'ambiguïté qui

62. Ibid., p. 456-457.

63. Ibid., p. 448.

64. J. D. Gould, «Hypothetical History», art. cit.

65. J. D. Gould, art. cit., p. 195-196. 
provient d'une spécification inadéquate des changements de croyances requis par la présupposition contrefactuelle ${ }^{66} \gg$.

La principale difficulté des raisonnements contrefactuels pour les historiens vient du fait qu'ils ne peuvent soumettre leurs contrefactuels à une expérimentation. En lieu et place de cette dernière, l'histoire économique peut cependant proposer des explications. Elle peut, sous certaines conditions, construire des quasi-lois en recourant à une approche hypothético-déductive. S'inspirant ici de l'argument de Redlich, Gould veut distinguer cette manière de faire de l'histoire, qu'il appelle «directe», de la méthode contrefactuelle, qui «prend pour point de départ une chose dont il est admis qu'elle est fausse ${ }^{67} »$. Mais ces deux approches sont, selon lui, complémentaires et il importe de bien déterminer leur domaine respectif de validité. L'usage du raisonnement contrefactuel est utile précisément - contrairement à ce qu'affirmait et pratiquait Fogel - lorsqu'il n'existe pas de généralisations robustes permettant de construire une hypothèse, par exemple lorsqu'il s'agit de déterminer les effets de la Révolution industrielle sur le niveau de vie britannique. Si cette recherche ne peut s'appuyer sur une généralisation, explique Gould, c'est parce que l'événement étudié est unique mais aussi et parce qu'il est complexe, ouvert : parce qu'il recouvre «un ensemble de processus liés les uns aux autres se répandant sur une vaste surface d'expérience humaine et [que] ses causes comme ses conséquences sont également nombreuses, compliquées et difficiles à démêler ${ }^{68} \gg$. L'approche contrefactuelle est donc intéressante dans la mesure où elle permet de simplifier et de fermer subitement des processus historiques complexes et ouverts, en déclarant arbitrairement leur inexistence et en mesurant ce qui aurait été perdu ou gagné par cette soustraction.

Une fois déterminé leur intérêt pour certains problèmes historiques, il reste à fixer les conditions du délicat maniement des contrefactuels. Comme pour Gerschenkron, les contrefactuels sont, selon Gould, mieux adaptés à des relations portant sur une période de court terme où situations réelles et contrefactuelles peuvent être comparées en supposant de nombreuses variables égales par ailleurs. Ensuite, il faut admettre que les raisonnements contrefactuels sont parfois impossibles à mettre en

66. Ibid., p. 196.

67. Ibid., p. 195.

68. Ibid., p. 199 
œuvre à la manière des raisonnements hypothético-déductifs classiques, parce qu'il n'est pas possible de savoir quels changements exacts opérer dans la réalité pour construire la proposition contrefactuelle. Ainsi, s'il s'agit d'imaginer une Grande-Bretagne sans Révolution industrielle pour saisir l'effet de cette dernière sur le niveau de vie des Britanniques, il faut pouvoir faire des hypothèses sur l'évolution de la population dans ce cours alternatif de l'histoire alors même que cette évolution peut elle-même dépendre du niveau de vie, c'est-à-dire de l'effet qu'il est question de calculer ${ }^{69}$. La difficulté ne vient pas ici du raisonnement contrefactuel en lui-même, mais du caractère trop imprécis du problème à résoudre. Pour qu'un raisonnement contrefactuel soit fructueux, il faut donc également que les événements sur lesquels il porte ne soient en définitive pas trop ouverts.

Gould s'interroge enfin sur les relations entre pratique du raisonnement contrefactuel et construction de relations causales. Même quand ils sont faisables et qu'ils respectent les conditions d'usage qui viennent d'être mentionnées, tous les raisonnements contrefactuels ne permettent pas, n'en déplaise à Fogel, d'établir des causalités. Il est nécessaire de différencier une forme de l'approche contrefactuelle où l'inférence testée est «forte», et une «forme faible» où l'approche contrefactuelle ne permet pas d'établir une relation causale, comme dans l'énoncé « Si Napoléon avait eu la bombe atomique, il n'aurait pas perdu la bataille de Waterloo ${ }^{70} \gg$. Il reste que même si un raisonnement de type contrefactuel ne permet pas d'établir une relation causale, il peut au moins permettre de démontrer que l'événement nié par la proposition contrefactuelle ne pouvait pas être une cause suffisante du phénomène étudié, ce qui, selon Gould, pourrait bien constituer l'une des fonctions les plus intéressantes de cette méthode. Le raisonnement contrefactuel ne saurait donc, pour Gould non plus, devenir l'unique manière d'approcher la causalité et l'explication en histoire économique, mais doit au contraire être subordonné à une exploration complète de la réalité, de ses causes et de ses conséquences. Avant de pratiquer les raisonnements contrefactuels, l'historien doit apprendre à distinguer entre des contextes où son emploi est intéressant et d'autres où il n'apporterait rien de bien passionnant à la connaissance historique. Dans la pratique de l'histoire, tout ne peut se

69. Ibid., p. 201-202

70. Ibid., p. 204-205. 
réduire à une affaire de différence entre ce qui a effectivement eu lieu et ce qui aurait pu être ${ }^{71}$.

Si Gould et Gerschenkron, à deux ans d'intervalle, n'ont donc pas exactement les mêmes critères afin de définir le domaine de pertinence des raisonnements contrefactuels en histoire, à la fois contre les praticiens de la nouvelle histoire économique - Fogel au premier chef, mais aussi Meyer et Conrad - et contre leurs nombreux détracteurs, leurs interventions ont cependant durablement déplacé le débat dans cette discipline. Après 1969, il ne s'agit plus, en effet, dans cette arène, de défendre ou de refuser l'approche contrefactuelle, mais de déterminer quels sont les emplois les plus justes de cette approche et dans quels cas elle est, ou bien la plus appropriée, ou bien la plus féconde. Ce déplacement de l'épicentre de la polémique est d'ailleurs entériné en 1970 par Fogel luimême, dans un article de bilan sur ce qu'il appelle alors, dans la revue History and Theory, «l'économétrie rétrospective ${ }^{72}$ ». Il y reconnaît d'abord que «certaines assertions contrefactuelles sont plus difficiles à résoudre que d'autres », comme celle, par exemple, qui viserait à déterminer l'effet du nez de Cléopâtre sur le cours de l'histoire, puisqu'on ne dispose pas de généralisations à propos de l'effet des tailles de nez sur les décisions politiques ${ }^{73}$. Plus fondamentalement, Fogel admet que la pertinence d'un énoncé contrefactuel dépend de plusieurs facteurs. Or la plupart de ceux qu'il énumère sans s'y attarder ont été mentionnés par les critiques qu'il a reçues les années précédentes, en particulier de la part de Gould et Gerschenkron.

Pris ensemble, ces deux chercheurs ont par ailleurs insisté sur le fait que les événements singuliers, limités dans le temps et relativement fermés sont certainement les plus recommandés pour le raisonnement contrefactuel. Enfin, en contestant les conceptions de la causalité qui accompagnait, chez Fogel et plusieurs autres «nouveaux» historiens, la pratique de la cliométrie et des contrefactuels, ils ont proposé que l'emploi de ces approches fasse désormais l'objet d'une analyse logique et surtout épistémologique plus poussée qu'elle ne l'était précédemment. Ainsi la deuxième moitié des années 1960 aux États-Unis est-elle marquée par

71. Ibid., p. 206-207.

72. R. W. Fogel, «Historiography and Retrospective Econometrics», History and Theory, 9/3 (1970), p. 245-264

73. Ibid., p. 262-263. 
deux phénomènes importants pour la conception des contrefactuels en histoire : une marginalisation du débat pro et contra au profit d'un exercice de définition du territoire empirique propre à ce mode de raisonnement; une mise en liaison, ténue mais effective, des débats entre historiens et de la discussion philosophique ouverte au milieu des années 1940 sur le même thème, discussion jusqu'alors cantonnée aux frontières de l'historiographie ${ }^{74}$.

L'étude de l'apparition puis de la circulation du mot «contrefactuel» et de ses dérivés dans le corpus savant permet donc d'isoler la période de constitution et de développement de ce qu'on a appelé la «nouvelle histoire économique » américaine, de la fin des années 1950 au début des années 1970, comme un moment décisif aussi bien pour l'identification que pour la pratique et la discussion du contrefactualisme en histoire. Connu des historiographes, particulièrement en histoire économique, ce moment n'avait pourtant pas fait l'objet d'une analyse spécifique dans les contributions contemporaines nombreuses portant sur l'intérêt des contrefactuels en histoire et en sciences sociales. L'enquête montre que l'élaboration, à partir du milieu des années 1940, d'un discours philosophique et logique sur les contrefactuels est, durant ce moment de la new economic history, un arrière-plan ignoré ou négligé des historiens, dans leurs usages du mot comme dans leurs controverses. Toutefois, du point de vue méthodologique et épistémologique, il est possible de distinguer assez nettement trois phases de ces dernières.

Jusqu'au début des années 1960, on assiste aux débuts d'une réflexion explicite sur les contrefactuels chez les historiens et les épistémologues

\footnotetext{
74. Une enquête parallèle à celle que nous avons conduite pourrait être menée au sujet des emplois du mot «contrefactuel» et des discussions sur les approches contrefactuelles en épistémologie de l'histoire proprement dite, terrain éventuel de rencontres entre philosophes et historiens. Pour la période considérée ici, les articles suivants font explicitement référence aux « contrefactuels » dans un débat plus général sur la scientificité de l'histoire alors polarisé par les prises de position respectives de Hempel et de Dray dans les années 1940 et 1950 : Walter Fales, «Historical Facts », Journal of Philosophy, 48/4 (1951), p. 85-94 ; Carey B. Joynt, Nicholas Rescher, «The Problem of Uniqueness in History ", History and Theory, 1/2 (1961), p. 150-162; John Lange, «The Argument from Silence», History and Theory, 5/3 (1966), p. 288-301 ; George G. S. Murphy, «On Counterfactual Propositions », History and Theory, 9/9 (1969), p. 14-38. La lecture de ces textes ne remet pas en cause le récit et l'analyse que nous avons proposés ici.
} 
de l'histoire. Ces énoncés sont d'emblée abordés dans une perspective structurée par plusieurs oppositions : entre science nomologique et compte rendu idiographique, explication et compréhension, entre déterminisme - marxiste notamment - et une histoire qui serait exclusivement descriptive. Que les différents contributeurs du discours sur les contrefactuels en histoire les tiennent pour possibles ou impossibles, ils leur semblent en tout cas aller dans le sens de l'introduction de perspectives nomologique et explicative dans la discipline - ces deux visées étant généralement tenues pour équivalentes. Chez certains, l'introduction d'hypothèses contrefactuelles pourrait constituer un moyen de ne pas abandonner l'horizon explicatif de l'histoire sans pour autant accepter le déterminisme, que celui-ci soit ou non mono-causal. Dans ce cadre, on se demande par exemple si l'histoire peut être alignée avec les sciences naturelles ou bien quel est le rôle des lois dans l'histoire.

Une deuxième phase du débat historien sur les contrefactuels commence à partir de 1965 et de la polémique entre Fritz Redlich et Robert Fogel. Il s'agit alors de discuter d'une nouvelle école historique dont le raisonnement contrefactuel est un aspect capital, même s'il n'est pas la seule dimension qui la définisse - ce dont témoigne par exemple le fait que cette école n'est pas encore qualifiée d'histoire contrefactuelle alors que cela sera parfois le cas dans la période ultérieure. L'essentiel de la controverse porte alors sur la question de l'observabilité des contrefactuels et de la testabilité des hypothèses contrefactuelles : les énoncés contrefactuels peuvent-ils être des hypothèses empiriques? Chez les nouveaux historiens comme Fogel, cela conduit à une reformulation positiviste de l'approche contrefactuelle, là où philosophes et historiens de la période précédente défendaient plutôt l'idée d'une utilité des abstractions et des fictions dans les sciences, c'est-à-dire la fécondité de l'imagination et de l'irréel. Sous l'impulsion principale de Fogel, qui ignore cette dimension soulignée par Redlich, son adversaire le plus franc, le débat se construit alors sur une opposition entre une histoire traditionnelle qui mobiliserait des raisonnements contrefactuels sans le savoir, et une histoire nouvelle, qui formule explicitement ses hypothèses et les teste de façon conséquente.

Dans la seconde moitié des années 1960 s'ouvre une autre période, où le débat se déplace vers une évaluation plus subtile des mérites du contrefactualisme, de ses domaines d'application privilégiés, et vers la question de savoir dans quels cas le raisonnement contrefactuel est ou non fécond en histoire. La démarche contrefactuelle est alors définie de 
manière plus précise par des observateurs extérieurs de la nouvelle histoire économique qui entendent en délimiter la pertinence et en fixer le mode d'emploi. Leur idée principale est que ce qui devrait intéresser l'historien qui utilise la démarche contrefactuelle n'est pas tant de vérifier une loi générale que d'imaginer ce qui se passerait dans tel et tel cas particuliers, au moyen éventuellement de généralités. On assiste, chez certains, à une remise en cause de l'idée, diffuse jusqu'alors, selon laquelle le recours aux hypothèses contrefactuelles s'appuierait sur la nature nomologique de la connaissance historique ou la confirmerait. À cela s'ajoute une tentative pour dire dans quels cas la démarche contrefactuelle est nécessaire, fructueuse, improductive ou absurde.

$\mathrm{Au}$ terme de ces trois phases, le terme «contrefactuel» a non seulement été introduit chez les historiens et accepté par une partie d'entre eux, mais son sens a changé. La pratique des raisonnements contrefactuels est désormais légitime, du moins pour certains, au titre de méthode historique et plus seulement d'exercice littéraire. Elle n'implique pas nécessairement d'avoir à choisir entre une conception de l'histoire comme récit soumis à l'épreuve de l'archive ou comme science nomologique visant à la généralité. Plus radicalement, le débat sur les contrefactuels entamé à partir des controverses autour de la nouvelle histoire économique américaine montre, dans sa dynamique même, que cette méthode et ce mode de raisonnement ouvrent un territoire nouveau pour une épistémologie de l'histoire dégagée de cette alternative entre histoire-récit et histoire-science. Quant à la question que posent les contrefactuels aux historiens, elle ne consiste plus à prendre leur parti ou à s'y opposer. À la fin des années 1960, il y a donc plutôt lieu de savoir quelle épistémologie peut soutenir cette pratique, pour quel type de problèmes historiques les épreuves contrefactuelles sont les plus fécondes et comment équilibrer raisonnements factuels et contrefactuels dans l'enquête et la restitution historiques.

Un nouveau chapitre de l'histoire du concept de contrefactuel en sciences humaines s'ouvre cependant en 1974 avec la publication d'un article de deux philosophes dans la principale revue ayant présenté la nouvelle histoire économique. Ils y mentionnent l'ouvrage du philosophe David Lewis paru l'année précédente sous le titre de Counterfactuals ${ }^{75}$.

75. T. A. Climo et P. G. A. Howells, «Cause and Counterfactuals», The Economic History Review, n.s., 27/3 (1974), p. 461-468. Voir aussi id., «Possible Worlds in Historical Explanation», History and Theory, 15/1 (1976), p. 1-20. 


\section{Labyrinthe, $n^{\circ} 39$}

Le lien jusqu'alors distendu voire inexistant entre discussions logiques, épistémologiques et historiographiques sur les contrefactuels y est resserré autour de la question de la causalité et des formes de l'explication historique. Il n'est plus question alors de contester l'histoire contrefactuelle pratiquée par la new economic history, ni les perspectives qu'elle a ouvertes pour les autres domaines de l'histoire et les autres sciences sociales. La controverse sur les contrefactuels en sciences humaines entre alors dans une autre phase où elle pose de nouveaux problèmes. 